\title{
RANDOMICITY CHARACTERISTICS OF FLASH EVAPORATION OF PURE WATER DROPLETS IN QUICK DEPRESSURIZATION PROCESS
}

\author{
Jian-Fu Zhao, ${ }^{1,2, *}$ Wang-Fang Du, ${ }^{3}$ \& Kai Li ${ }^{1,2}$ \\ ${ }^{1}$ Key Laboratory of Microgravity, Institute of Mechanics, Chinese Academy of Sciences, Beijing \\ 100190, China \\ ${ }^{2}$ School of Engineering Science, University of Chinese Academy of Sciences, Beijing 100049, \\ China \\ ${ }^{3}$ State Nuclear Power Technology Corporation Research E Development Center, Beijing 102209, \\ China \\ *Address all correspondence to Jian-Fu Zhao, E-mail: jfzhao@imech.ac.cn
}

\begin{abstract}
A series of experiments on the flash evaporation-freezing process of pure water droplets released into vacuum have been performed in order to reveal the influences of randomicity, with particular attention being paid to the random characteristics of the relevant sub-processes. It was clearly observed that the nucleation time is a random, independent variable that drastically affects the nucleation temperature and supercooling. Both the nucleation temperature and supercooling are not dependent on the terminal environmental pressure. They exhibit similar means and standard deviations at different terminal environmental pressures. The freezing time is independent of the nucleation time, but exhibits an obvious dependence on the terminal environmental pressure and drop diameter. The freezing temperature exhibits a much more complicated characteristic, which may be determined by the real atmosphere around the droplets. The measured quantitative characteristics will be helpful in developing a model to predict the flash evaporation-freezing process of pure water droplets during quick depressurization.
\end{abstract}

KEY WORDS: flash evaporation, randomicity, nucleation, freezing, supercooling, quick depressurization

\section{INTRODUCTION}

Flash evaporation of liquid droplets may occur when the system pressure is suddenly dropped to a value far below the saturation pressure corresponding to the liquid temperature. It is important in the fundamental view to study the physical processes accompanying the phenomenon, such as instant boiling, phase transitions on surfaces and inside droplets, disintegration on droplets, etc. On the other hand, studying this phenomenon is also of great interest, in particular, for space technology. Potential uses include liquid droplet radiators, liquid stream orientation thrusters, liquid drainage devices, and so on. Thus, studying the flash evaporation process of liquid droplets during quick depressurization has attracted more and more attention with the rapid progress of aerospace engineering (Zhao et al., 2011). Moreover, flash evaporation of liquid droplets can also be used widely in a multitude of industrial and technological fields, such as refrigeration (Shin et al., 2000; Satoh et al., 2002), artificial snow manufacture (Kim et al., 2001; Strub et al., 2003), seawater desalination (Darwish and Al-Najem, 2000; Liu et al., 2009; Liu, 2010), food preservation (Hindmarsh et al., 2004; Nam and Song, 2007), and so on.

The objective of this study is to investigate experimentally the flash evaporation process of pure water droplets released into vacuum, particularly the detailed characteristics of the sub-processes relevant to quick or flash evaporation and freezing during quick depressurization. Generally, liquid droplets released suddenly in a low-pressure 
environment from the atmosphere will become superheated due to the environmental pressure becoming lower than the saturation pressure corresponding to the initial droplet temperature. The surplus sensible heat is then released in the form of latent heat, which causes rapid—or flash-evaporation on the droplet surface. Meanwhile, the droplet temperature decreases quickly. Furthermore, this may eventually lead to freezing of the droplets. Thus, droplet evaporation and freezing during quick depressurization is a coupled heat and mass transfer process accompanied by phase change.

Many studies have thus far investigated pure water droplet evaporation under reduced pressure (Owen and Jalil, 1991; Shin et al., 2000; Kim et al., 2001; Satoh et al., 2002; Hindmarsh et al., 2003, 2004; Strub et al., 2003; Liu et al., 2007; Du et al., 2012; Zhang et al., 2012; Cheng et al., 2015). Owen and Jalil (1991), among many others, recorded the transient pressure and droplet temperature during flash evaporation by using the droplet hanging method. In followup studies, most researchers have adopted similar methods. Furthermore, Hindmarsh et al. (2004) investigated shape change by using magnetic resonance imaging (MRI). Generally, the flash evaporation and freezing process of a liquid droplet during quick depressurization (shown in Fig. 1) can be classified into the following five distinct stages:

1. Liquid cooling by flash evaporation: The liquid droplet is cooled from its initial state to a temperature below the equilibrium freezing point.

2. Nucleation: Crystal nucleation occurs spontaneously, which is driven by sufficient supercooling.

3. Recalescence: Rapid kinetic crystal growth from crystal nuclei is driven by supercooling, which liberates latent fusion heat and causes an abrupt temperature rise.

4. Freezing of the liquid droplet: Further growth of the solid phase governed by the rate of heat transfer to the environment from the droplet continues until the droplet is completely frozen.

5. Solid cooling by sublimation: The temperature of the frozen droplet reduces gradually to a steady-state value under the effects of convection and conduction in the ice (due to the temperature variation along the droplet radius) and mass transfer by sublimation of ice on the surface.

In Fig. 1, some characteristic parameters relevant to the process are also shown, including the nucleation time $\left(\tau_{n}\right)$ and corresponding temperature of the liquid droplet $\left(t_{n}\right)$, and the freezing temperature $\left(t_{f}\right)$ and corresponding duration of supercooling $\left(\Delta T_{\text {sup }}\right)$, and freezing $(\Delta \tau)$. To determine these characteristic parameters is the main aim in many of the theoretical studies on this subject in the literature. Shin et al. (2000) and Kim et al. (2001) analyzed the heat and mass transfer process of a water droplet during rapid evaporation by using the diffusion control model.

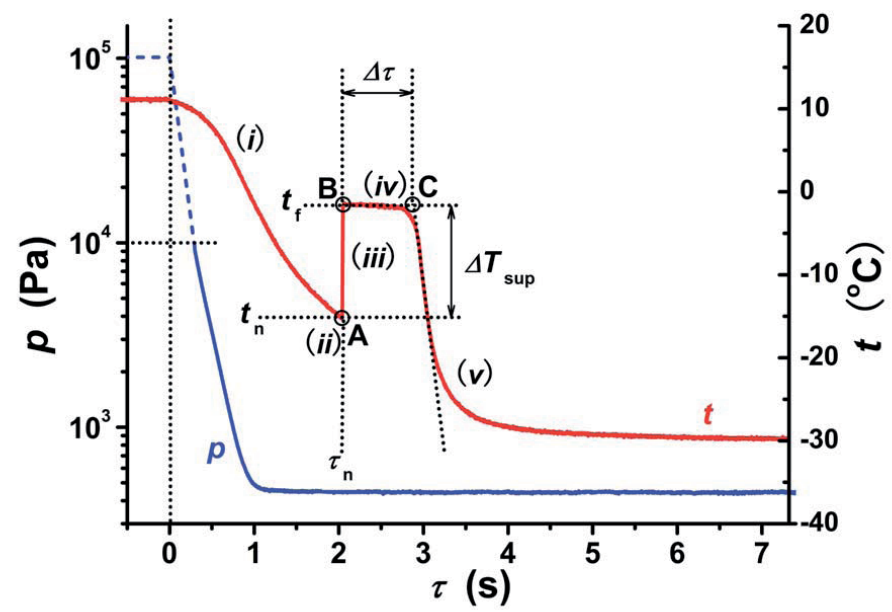

FIG. 1: Typical flash evaporation and freezing process of a water droplet during quick depressurization 
Zhao et al. (2011) and Zhang et al. (2012) established independently a lumped thermodynamic model to predict the droplet temperature variation during the flash evaporation and freezing process. Cheng et al. (2015) developed a comprehensive mathematical model of vacuum flash evaporation cooling of a water droplet based on the film controlled evaporation model and numerical analysis of the droplet temperature and diameter after evaporation. There are also many theoretical models and numerical analyses for the cases of multi-component fluid droplets (Liu et al., 2016) and droplets containing colloidal matter (Nešić and Vodnik, 1991). Since they are not within the scope of the present study, interested readers are referred to Liu et al. (2016) and references therein.

It is well known that the sub-process of nucleation exhibits an obvious randomicity due to the nature of the metastable state of a supercooled liquid. However, to date, there have been few theoretical studies on the randomicity of the sub-process of nucleation and its effect. Thus, in order to reveal the influences of nucleation randomicity, the flash evaporation and freezing process of pure water droplets released into vacuum is investigated experimentally in the present study. Particularly, the quantitative characteristics of the process are reported and discussed in detail, which can provide a database for further theoretical development in relation to this phenomenon.

\section{EXPERIMENTAL SYSTEM}

A schematic diagram of the experimental system is shown in Fig. 2 (Du et al., 2012, 2013). The system consisted of several parts: test vessel, vacuum system, data collection system, and high-speed photography system. The test vessel was made of aluminum with glass windows on two opposite sides. A thin T-type thermocouple (outside diameter $=$ $100 \mu \mathrm{m}$ ) was located inside the vessel to hang a liquid droplet and to simultaneously measure the temperature change of the droplet. The location of the measuring thermocouple junction was adjusted to be at the center of the water droplet. The thermocouple was thin enough to reduce the heat coming into the water from its surroundings and then to make the water freeze effectively by evaporating the water itself. The relevant temperature transmitter (with a range of $-100^{\circ}$ to $100^{\circ}$ and an uncertainty of $0.5^{\circ}$ ) measured the drop temperature at the thermocouple junction. A DMP331 absolute pressure transducer (BD-SENSORS) with a range of 0-0.01 MPa, uncertainty of $0.175 \%$ full scale, and response time $<5 \mathrm{~ms}$ was connected to the test vessel to measure the pressure change during the experiment.

The vacuum system included a vacuum pump, vacuum chamber, and solenoid valve. The vacuum pump, with a pumping rate of $8 \mathrm{~L} / \mathrm{s}$ and final vacuum of $6 \times 10^{-2} \mathrm{~Pa}$, was connected to the vacuum chamber. The volume of the vacuum chamber was $1 \mathrm{~m}^{3}$, about 800 times bigger than that of the test vessel. Therefore, it can maintain constant pressure in the vessel during the experiment. A solenoid valve (inside diameter $=25 \mathrm{~mm}$ ) between the test vessel and the vacuum chamber was used to speed up the depressurization process. The performance test of the

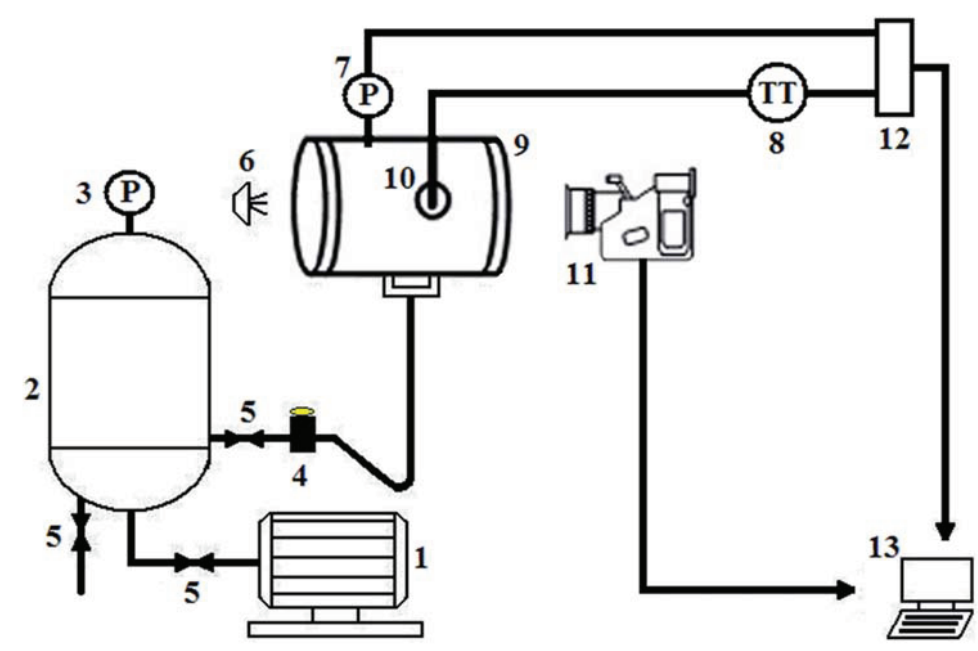

FIG. 2: Schematic diagram of the experimental system 
experimental system verified that the pressure inside the test vessel can be reduced within a period no longer than $1 \mathrm{~s}$ from atmospheric pressure to the designed terminal environmental pressure (Du et al., 2012, 2013).

In the present experiment, pure distilled water was used as the test material. First, a water droplet was hung in the test vessel with the thin thermocouple. After the test vessel was sealed, the air inside the test vessel was quickly evacuated by opening the solenoid valve connected to the vacuum chamber. The liquid droplet temperature dropped quickly, which was caused not only by the expanding air around it, but also by the quick evaporation on its surface. After the temperature exceeded some low limit, the droplet was frozen from the surface. The liquid was transformed into a solid state, releasing the latent heat. Then, the temperature rose to a value near the freezing point, and was maintained until the whole droplet was completely frozen. Finally, its temperature dropped again due to surface sublimation.

A high-speed AOS Vitcam CTC camera with a record rate of 500 frames per second at $1280 \times 1024$ pixels was used to record the shapes of the liquid droplets during the experiments. The exposure time was adjusted automatically based on the lighting condition, which was provided by a compact fluorescent light bulb. The field of view was adjusted to ensure that the droplets were in the central position and not less than one-quarter of the entire field of view. The droplet diameter was obtained from the recorded image of its shape by assuming a revolution body for the droplet. Furthermore, a signal fed back from the driving circuit of the solenoid valve was used to trigger the highspeed camera. All data including the outputs from the pressure transducer and the temperature transmitter, as well as the feedback signal, were recorded by a DATAQ DI-710 data collection system. Thus, a good corresponding relation could be obtained between the measured temperature and pressure data and the recorded images of the droplet shape.

\section{RESULTS AND DISCUSSION}

A series of experiments were conducted at three nominal values of the terminal environmental pressure, namely, 450, 600 , and $1000 \mathrm{~Pa}$. The ambient temperature was kept constant $\left(\sim 7^{\circ}\right)$ in the test vessel, while the initial diameter of droplets was 1.4-2.1 mm. Typical histories of the temperature for five droplets and the pressure inside the test vessel during quick depressurization are shown in Fig. 3 under the terminal environmental pressure of $1000 \mathrm{~Pa}$. Careful analyses of the experimental data showed that the dispersion of the nucleation time cannot be explained by the diversity of other factors, such as the initial diameter, initial temperature, environmental temperature, and so on (Du et al., 2012). Thus, it is clearly shown that the nucleation time is a random variable. The randomicity signifies the dispersion of the nucleation time at the same condition of the initial temperature, terminal environmental pressure, and so on. Therefore, the nucleation time should be considered to be an independent, or input, variable—not a dependent, or output, variable —in further development of the phenomenological model of the process.

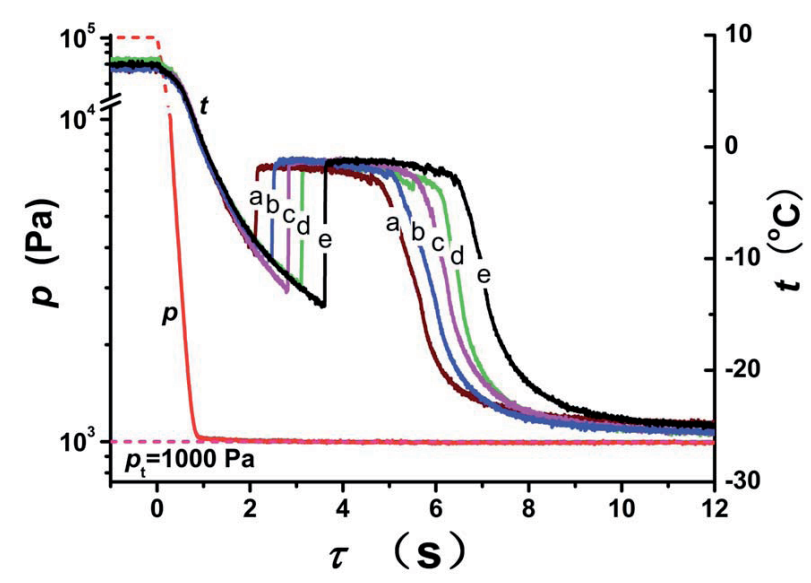

FIG. 3: Typical histories of the temperature of the droplet and of the pressure inside the test vessel during quick depressurization at the terminal environmental pressure of $1000 \mathrm{~Pa}$ 
Histograms of the nucleation time are shown in Fig. 4 for three different terminal environmental pressures. Each histogram was obtained based on a database with more than 60 experimental data. Generally, the nucleation time increases with an increase in the terminal environmental pressure. However, only the terminal environmental pressure cannot determine the nucleation time. Figure 4 shows that dispersion of the nucleation time also increases with an increase in the terminal environmental pressure. The difference between the saturated pressure relevant to the initial temperature of liquid droplets and the terminal environmental pressure represents the driving force behind evaporation on the liquid surface and/or sublimation on the ice surface of the droplets. This decreased with an increase in the terminal environmental pressure at nearly constant initial temperature of the liquid droplets in the present study, resulting in the two observations previously mentioned.

This randomicity may be caused by the following facts. Supercooled water is in a thermodynamic metastable state, which may be strongly affected by external perturbations, such as the release of dissolved air in water, shaking caused by air flow, evaporation of water on the fine wire of the thermocouple inside the droplets, and so on. These external perturbations are not deterministic but random, which may cause the random occurrence of the nucleation of the freezing sub-process during quick depressurization.

As shown in Fig. 3, obvious dependences of nucleation temperature $t_{n}$ and supercooling $\Delta T_{\text {sup }}$ on nucleation time $\tau_{n}$ are observed. This can also be observed more obviously in Fig. 5, for example, at the terminal environmental pressure of $1000 \mathrm{~Pa}$. These two parameters vary linearly with the nucleation time: nucleation temperature $t_{n}$ decreases linearly with an increase in nucleation time $\tau_{n}$, while supercooling $\Delta T_{\text {sup }}$ increases. The reason for the dependence of the nucleation temperature on the nucleation time can be explained by the following fact. The process of liquid cooling follows a similar locus at the same terminal environmental pressure. Thus, the earlier the nucleation occurs, the smaller is the observed decrease in the droplet temperature. Then, the nucleation temperature is higher and the supercooling is larger.

Figure 6 shows histograms of the supercooling at three different terminal environmental pressures. It is obvious that supercooling is independent of the terminal environmental pressure; namely, almost the same mean and standard deviation values are observed at different terminal environmental pressures.

It should be pointed out here that supercooling is calculated based on the difference between the freezing and nucleation temperatures. Figure 7 shows histograms of the freezing temperature at three terminal environmental pressures. Generally, the freezing temperature is also independent of the terminal environmental pressure. Values slightly smaller than $0^{\circ}$ were observed for the freezing temperature in most of the cases studied in the present experiments, which is different from the popular belief. Furthermore, no controllable factor responsible for scattering of the data was found in the present experiment.

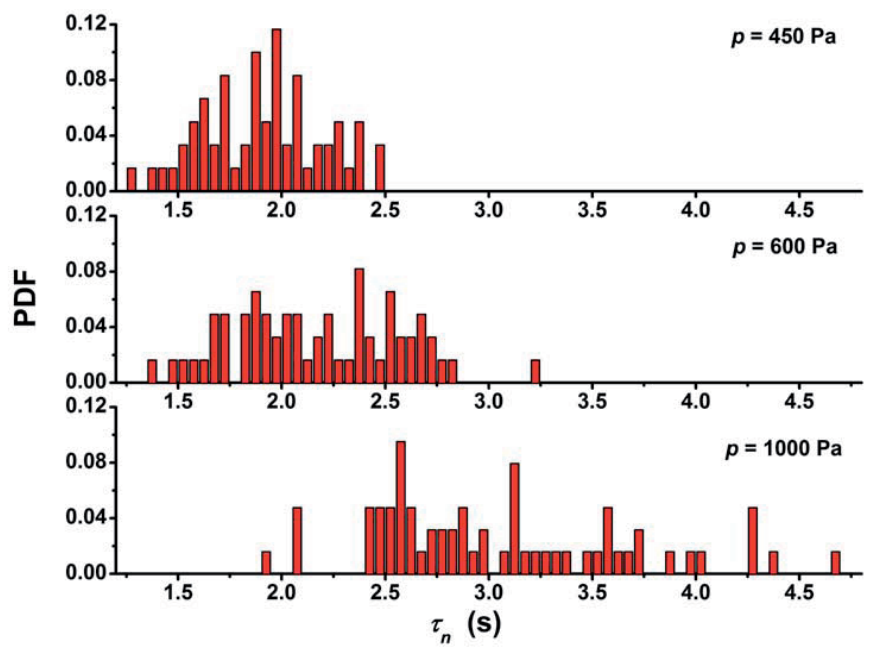

FIG. 4: Histograms of the nucleation time at different terminal environmental pressures

Volume 4, Issues 2-3, 2016 


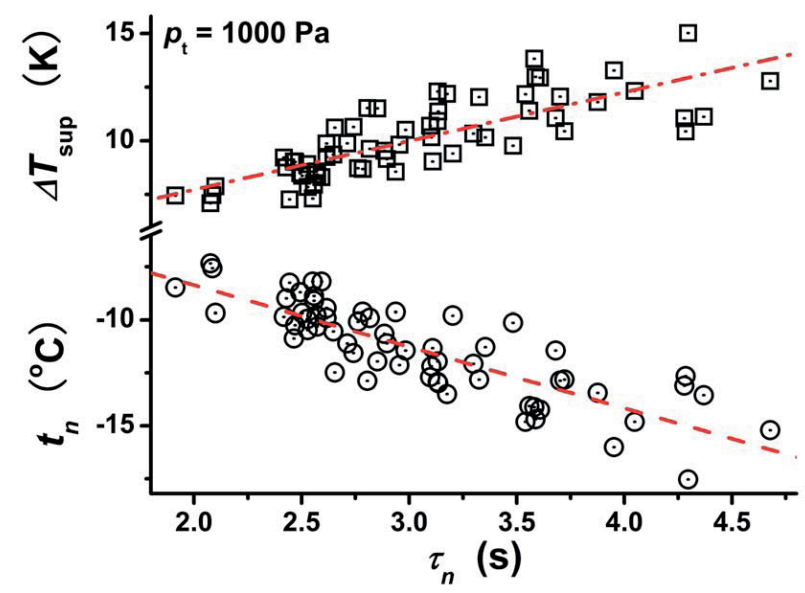

FIG. 5: Dependences of nucleation temperature and supercooling on the nucleation time at the terminal environmental pressure of $1000 \mathrm{~Pa}$

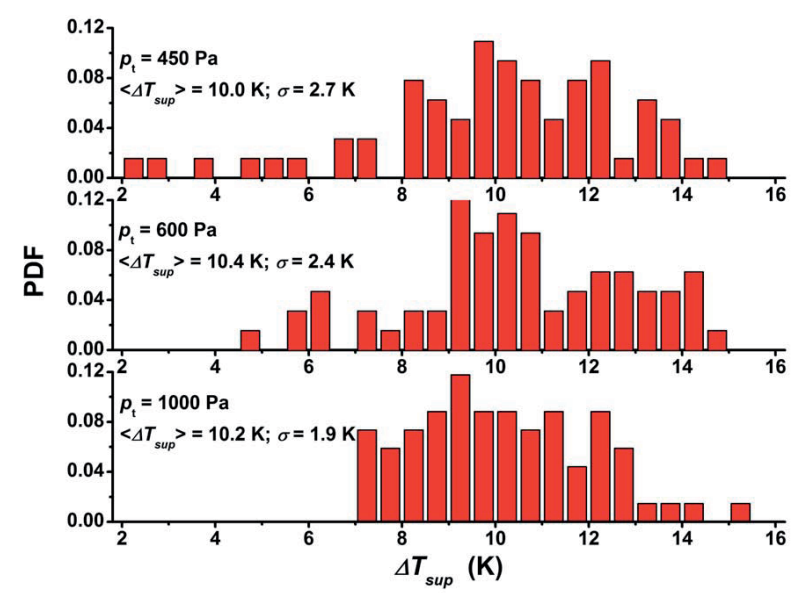

FIG. 6: Histograms of supercooling at different terminal environmental pressures

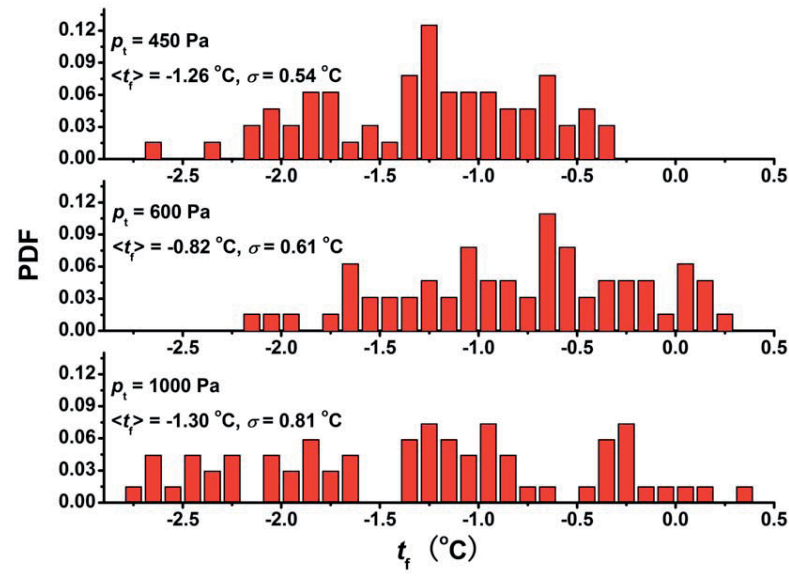

FIG. 7: Histograms of the freezing temperature at different terminal environmental pressures

Based on the measured droplet temperature and vessel pressure, the thermodynamic process can be plotted formality in a $p$ - $t$ chart (as shown in the scatter diagram in Fig. 8) for the flash evaporation-freezing process of water droplets during quick depressurization. Here, point $\mathrm{P}_{1,2}$ indicates the measured, or superficial, initial states by using the vessel or total pressure and the initial temperature for the two experimental runs shown in Fig. 8. For the case in which $p_{t}>611 \mathrm{~Pa}$ (the pressure at the triple point of water), the path shown as triangles, does not cross equilibrium line $O C$ between the water and vapor but crosses equilibrium line $O B$ between the water and ice. Based on the same observation, Liu (2010) oppugned the occurrence of flash evaporation in this case. However, the real thermodynamic process in the $p-t$ chart should be plotted based on the vapor partial pressure and its temperature because freezing usually occurred on the surface of the droplets, which is immersed in an atmosphere containing vapor and non-condensable air.

However, it is very difficult to measure the vapor partial pressure inside the test vessel during quick depressurization. Here, some possible paths are shown as dashed lines in Fig. 8. The real initial state for water near the droplet's 


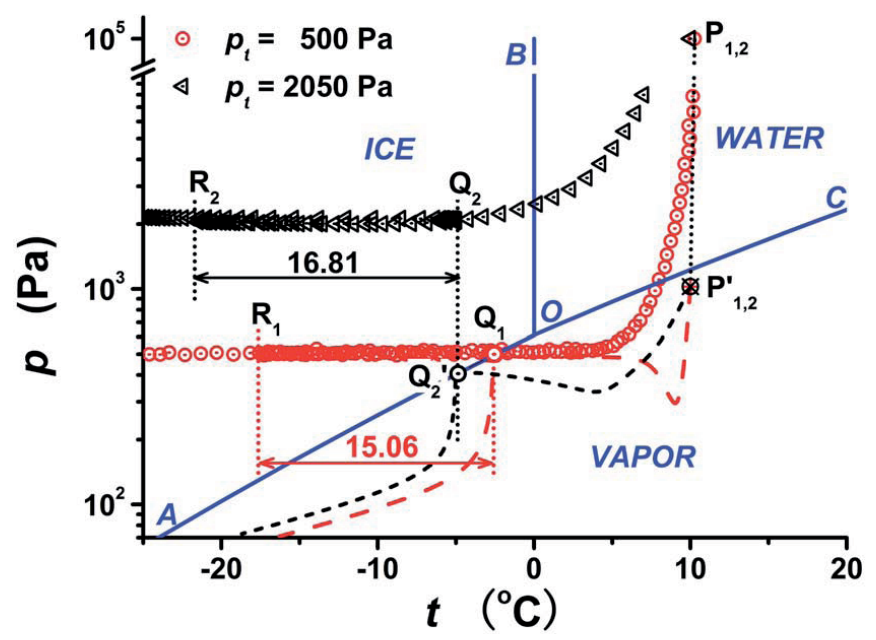

FIG. 8: Thermodynamic process of flash evaporation-freezing of water droplets during quick depressurization at different terminal environmental pressures

surface is not point $\mathrm{P}_{1,2}$ but point $\mathrm{P}_{1,2}^{\prime}$, which is located in the vapor region, reflecting the non-saturation of vapor in the atmosphere. This is based on the observation that the temperature of the droplet usually exhibits a slight decrease in the beginning of experiments. As soon as the solenoid valve is opened, gases inside the test vessel are evacuated quickly into the vacuum chamber, resulting in a quick decrease of vapor partial pressure and then flash evaporation of water on the droplet surface. The latter may lead to an increase in vapor content and then an increase in the ratio of the vapor partial pressure to the total pressure inside the test vessel. The lower the terminal environmental pressure, the stronger is the occurrence of flash evaporation of the water on the droplet surface, and the greater is the increase in vapor content and the ratio of the vapor partial pressure to the total pressure exhibited inside the test vessel. Once the nucleation occurs, part of the water is transformed into ice and latent heat is released to the other part of the water, resulting in a quick increase in the temperature of the droplet. This is called recalescence.

At the end of the recalescence sub-process, the temperature may be determined by the equilibrium between the vapor and ice (line $O A$ ). Thus, the freezing temperature will be determined by the intersection between its real path and vapor-ice equilibrium line $O A$. Then, although the terminal environmental pressure is higher than that of the triple point, as shown by path $\mathrm{P}_{2} \mathrm{Q}_{2} \mathrm{R}_{2}$ in Fig. 8, the freezing temperature relevant to point $\mathrm{Q}_{2}^{\prime}$ is obviously lower than that determined by the intersection between water-ice equilibrium line $O B$. This is also the reason why supercooling is defined as the difference between the freezing and nucleation temperatures. For the case of lower terminal environmental pressure, as shown by path $\mathrm{P}_{1} \mathrm{Q}_{1} \mathrm{R}_{1}$ in Fig. 8, the larger driving force behind evaporation on the liquid surface leads to larger vapor content and corresponding vapor partial pressure during the sub-process of liquid cooling by flash evaporation. Thus, point $\mathrm{Q}_{1}^{\prime}$, determined by the intersection between its real path and vapor-ice equilibrium line $O A$, is near point $\mathrm{Q}_{1}$.

As soon as the ice shell appears, the vapor production rate will decrease drastically from sublimation on the ice surface compared with that from evaporation on the liquid surface because of the greater latent heat needed for the ice-vapor change than for the liquid-vapor change, which is caused by the more stable structure of the molecule arrangement in ice than in water. The possible paths then turn downward, moving in the vapor region and providing the driving force, namely, the difference between the ice temperature and the corresponding saturated temperature at the same vapor partial pressure. The difference will approach a certain fixed value to maintain a balance between the heat absorbed by sublimation and that transferred from the ambient environment.

Figure 9 shows histograms of the freezing time at different terminal environmental pressures. It can be clearly observed that the freezing time increases with an increase in the terminal environmental pressure despite the randomicity. This was straightforward, based on the heat balance analysis. As previously mentioned, the lower the terminal envi- 


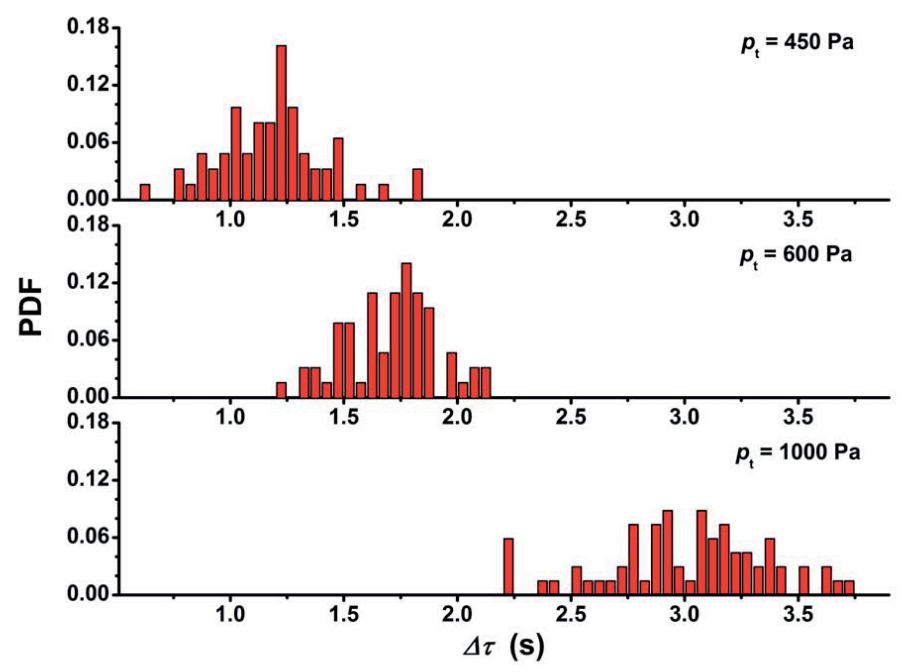

FIG. 9: Histograms of the freezing time at different terminal environmental pressures

ronmental pressure, the stronger is the occurrence of sublimation on the frozen droplet surface. Then, more heat can be transferred and less time is needed for the freezing stage.

Furthermore, an obvious dependence of the freezing time on the initial droplet diameter was also observed (see Fig. 10). A roughly linear increase in the freezing time on the initial droplet diameter was observed for each terminal environmental pressure. This was also straightforward, based on the heat balance analysis. A larger droplet contains a large quantity of water. Then, more latent heat needs to be released to make it completely freeze, and a correspondingly longer duration of time is observed for the freezing stage.

\section{CONCLUSIONS}

Flash evaporation of liquid droplets may occur when the system pressure is suddenly dropped to a value far below the saturation pressure corresponding to the liquid temperature. Heat absorbed during flash evaporation on the liquid surface may lead to freezing of the remaining liquid water. It is well known that the nucleation for freezing process

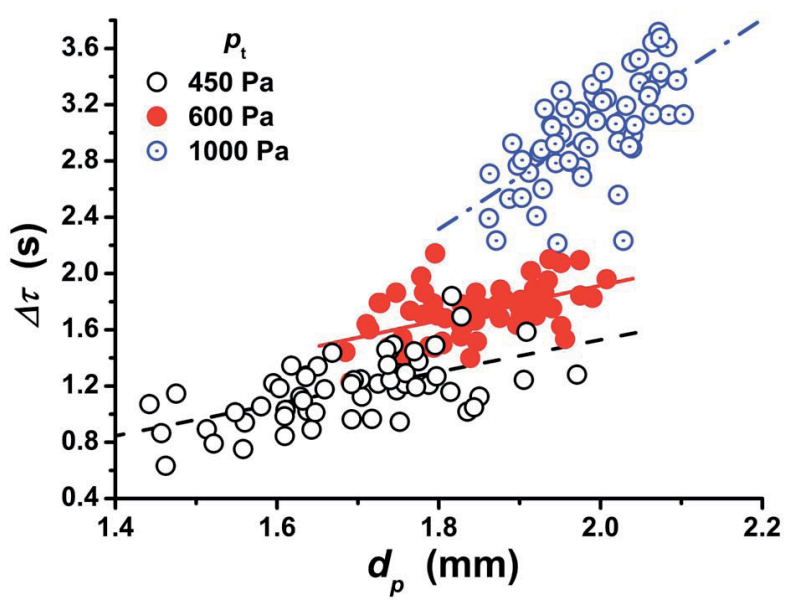

FIG. 10: Dependence of freezing time on initial droplet diameter at different terminal environmental pressures 
exhibits an obvious randomicity due to the nature of the metastable state of a supercooled liquid. Understanding the randomicity of the nucleation and its influence will be helpful in revealing the mechanism underlying the process of flash evaporation and freezing during rapid depressurization, and then for developing a theoretical and more physical model in relation to this phenomenon.

A series of experiments on the flash evaporation-freezing process of pure water droplets released into vacuum were performed in the present study, with particular attention being paid to the random characteristics of the relevant sub-processes. It was clearly observed that the nucleation time is a random, independent variable. The nucleation time more drastically affects the nucleation temperature and supercooling than the terminal environmental pressure. The freezing temperature exhibits a much more complicated characteristic. The influences of non-condensable gases may be one of the reasons for this complexity, and thus it may be determined by the real atmosphere around the droplets. This is independent of the nucleation time, but exhibits an obvious dependence on the terminal environmental pressure and drop diameter. The reported data on the statistical characteristics can be used to reveal the mechanism underlying the process and to further develop a phenomenological model of the process.

\section{ACKNOWLEDGMENT}

This work was supported partly by the Knowledge Innovation Program of the Chinese Academy of Sciences.

\section{REFERENCES}

Cheng, W. L., Chen, H., Hu, L., and Zhang, W. W., Effect of droplet flash evaporation on vacuum flash evaporation cooling: Modeling, Int. J. Heat Mass Transfer, vol. 84, pp. 149-157, 2015.

Darwish, M. A. and Al-Najem, N. M., Energy consumption by multi-stage flash and reverse osmosis desalters, Appl. Therm. Eng., vol. 20, no. 5, pp. 399-416, 2000.

Du, W. F., Li, K., Wang, S. F., and Zhao, J. F., Flashing liquid jets in low-pressure environment, Interfacial Phenomena Heat Transfer, vol. 1, no. 2, pp. 173-180, 2013.

Du, W. F., Zhao, J. F., and Li, K., Experimental study on thermal-dynamical behaviors of liquid droplets during quick depressurization, J. Eng. Thermophys., vol. 33, no. 8, pp. 1349-1352, 2012.

Hindmarsh, J. P., Buckley, C., Russell, A. B., Chen, X. D., Gladden, L. F., Wilson, D. I., and Johns, M. L., Imaging droplet freezing using MRI, Chem. Eng. Sci., vol. 59, pp. 2113-2122, 2004.

Hindmarsh, J. P., Russel, A. B., and Chen, X. D., Experimental and numerical analysis of the temperature transition of a suspended freezing water droplet, Int. J. Heat Mass Transfer, vol. 46, pp. 1199-1213, 2003.

Hindmarsh, J. P., Russell, A. B., and Chen, X. D., Experimental and numerical analysis of the temperature transition of a freezing food solution droplet, Chem. Eng. Sci., vol. 59, pp. 2503-2515, 2004.

Kim, B. S., Shin, H. T., Lee, Y. P., and Jurng, J., Study on ice slurry production by water spray, Int. J. Refrig., vol. 24, no. 2, pp. 176-184, 2001.

Liu, L., Study on mechanism of phase change heat and mass transfer of a droplet during reduced pressure process and in low pressure environment, Ph.D. Thesis, Xi'an Jiaotong University, Xi'an, Shaanxi, China, 2010.

Liu, L., Bi, Q. C., and Li, H. X., Experimental investigation on flash evaporation of saltwater droplets released into vacuum, Microgravity Sci. Technol., vol. 21, no. 1, pp. 255-260, 2009.

Liu, L., Liu, Y., Mi, M., Wang, Z., and Jiang, L., Evaporation of a bicomponent droplet during depressurization, Int. J. Heat Mass Transfer, vol. 100, pp. 615-626, 2016.

Liu, W. M., Bi, Q. C., Liu, L., and Ji, Y. Q., Study on the shape and temperature variations within droplet in low pressure environment, J. Eng. Thermophys., vol. 28, no. 6, pp. 957-960, 2007.

Nam, J. H. and Song, C. S., Numerical simulation of conjugate heat and mass transfer during multi-dimensional freeze drying of slab-shaped food products, Int. J. Heat Mass Transfer, vol. 50, pp. 4891-4900, 2007.

Nešić, S. and Vodnik, J., Kinetics of droplet evaporation, Chem. Eng. Sci., vol. 46, no. 9, pp. 527-537, 1991.

Owen, I. and Jalil, J. M., Heterogeneous flashing in water drops, Int. J. Multiphase Flow, vol. 17, no. 5, pp. 653-660, 1991. 
Satoh, I., Fushinobu, K., and Hashimoto, Y., Freezing of a water droplet due to evaporation-heat transfer dominating the evaporation-freezing phenomena and the effect of boiling on freezing characteristics, Int. J. Refrig., vol. 25, no. 2, pp. 226$234,2002$.

Shin, H. T., Lee, Y. P., and Jurng, J., Spherical-shaped ice particle production by spraying water in vacuum chamber, Appl. Therm. Eng., vol. 20, no. 5, pp. 439-454, 2000.

Strub, M., Jabbour, O., Strub, F., and Bédécarrats, J. P., Experimental study and modelling of the crystallization of a water droplet, Int. J. Refrig., vol. 26, pp. 59-68, 2003.

Zhang, X. L., Wang, W., Li, Z. W., Li, X. F., Yu, M., and Liu, X. W., Modeling and experimental research of crystallization process of static droplet, J. Eng. Thermophys., vol. 33, no. 8, pp. 1419-1422, 2012.

Zhao, K. X., Zhao, J. F., Chen, S. L., and Du, W. F., Thermodynamics of flashing/freezing process of a droplet in vacuum, Chin. J. Space Sci., vol. 31, pp. 57-62, 2011. 